\title{
FACTORES SOCIOCULTURALES Y DEMOGRÁFICOS QUE INCIDEN EN LA ADOPCIÓN DE UN SERVICIO FINANCIERO INNOVADOR
}

\section{Sociocultural and Demographic Factors That Impact on the Adoption of an Innovative Financial Service}

Revista Trama

Volumen 9, número 2

Julio - Diciembre 2020

Páginas 115-165

ISSN: 1659-343X

https://revistas.tec.ac.cr/trama

Oscar Andrés Acuña Muñoz'

Fecha de recepción: 24 de julio, 2020

Fecha de aprobación: 1 de diciembre, 2020

Acuña, O. (2020). Factores socioculturales y demográficos que inciden en la adopción de un servicio financiero innovador. Trama, Revista de ciencias sociales y humanidades, Volumen 9, (2), Julio-Diciembre, págs. 115-165.

DOI: https://doi.org/10.18845/tramarcsh.v9i2.5570

1. Profesor del énfasis en Finanzas del programa de Maestría en Dirección de Empresas del Instituto Tecnológico de Costa Rica. Sede Inter-universitaria. Alajuela, Costa Rica. Representante Legal de Regional Leasing Services S.A. 


\section{RESUMEN}

El objetivo de este estudio es analizar la incidencia de factores tanto socioculturales como demográficos, que se involucran en la toma de decisión de los individuos al adoptar servicios financieros innovadores. Para lograr el objetivo se utiliza como base la Teoría de Acción Planificada y la Teoría de la Difusión de las Innovaciones. Se realiza la aplicación de un cuestionario como instrumento de medición en una población de 321 personas con edades entre los 18 a más 60 años. El análisis de los datos recolectados determina que la percepción de utilidad es el factor que más influye en la toma de decisiones de las personas por adoptar servicios financieros innovadores, por lo que se hace necesario enfatizar en los beneficios de los servicios financieros para incrementar su nivel de adopción y con esto contribuir al desarrollo de los sistemas financieros de los países.

Palabras clave: adopción; demografía; innovación; servicios financieros; socio cultura.

\section{ABSTRACT}

The objective of this study is to analyze the incidence of both sociocultural and demographic factors, which are involved in the decision-making process of individuals when adopting innovative financial services. To achieve the objective, the Theory of Planned Action and the Theory of Diffusion of Innovations are used as a basis. A questionnaire was applied as a measurement instrument in a population of 321 people with ages between 18 and over 60 years. The analysis of the collected data determines that factors such as the perception of utility are the ones that most directly influence people's decision-making to adopt innovative financial services, so it is necessary to emphasize the benefits of financial services to increase its level of adoption and thereby contribute to the development of the countries' financial systems.

Key words: adoption; demographics; financial services; innovations; socio-culture. 


\section{INTRODUCCIÓN}

Tradicionalmente se considera que la innovación es esencial para promover el crecimiento y desarrollo económicos (Schumpeter, 1942). Por lo anterior, es relevante entonces, buscar alternativas financieras innovadoras que permitan cumplir las expectativas lógicas de un mercado competitivo en donde interactúen libremente la oferta y la demanda de servicios financieros y éstos a su vez, cumplan con el objetivo primordial, no solo de satisfacer a sus demandantes, sino también, se considera que la innovación en las empresas de servicios es necesaria y estratégica debido a la importancia que los servicios tienen en la economía global y en el desarrollo social de los países y especialmente en Costa Rica, dada la situación que se ha vivido en los últimos años, en donde la crisis económica toma aún más fuerza con la aparición de la pandemia del COVID-19.

Ahora bien, es importante entender que en la sociedad los individuos pueden decidir con base en su sentimiento emocional y su pensamiento racional ante diferentes situaciones, pero, por otra parte, el entorno social puede inducir a que la decisión individual esté basada en su sentimiento o en su pensamiento (Cosentino, Azzollini, Depaula y Castillo, 2016). Es por esta razón, que no solo es necesario fortalecer los procesos de innovación y emprendedurismo financiero, sino también es indispensable conocer los aspectos que inciden en los procesos de toma de decisión de las personas, una vez que lleven a cabo sus procesos de interacción con otros individuos en los ambientes sociales a los que normalmente se ven expuestos, ya sea en el trabajo, la familia, los amigos y las propias costumbres.

Dado lo anterior existe poca evidencia en la literatura, orientada a explicar el comportamiento de los individuos en los procesos de adopción de servicios propiamente financieros, pues se logra identificar información relevante al tema de adopción de innovación principalmente orientado a aspectos tecnológicos, entre otros, pero no se encuentra evidencia, que haga una relación para determinar cómo la sociedad y sus costumbres inciden directa o indirectamente en estos procesos de adopción financiera. 
En este artículo se van a mostrar los resultados de los estudios de aquellas variables que afectan la toma de decisiones de las personas para efectos de llevar a cabo un proceso de adopción de servicios financieros innovadores. Es claro que muchos individuos aceptan la existencia de nuevos servicios, no obstante, no todos los individuos adoptan estos servicios, para su uso cotidiano. En el proceso de adoptar un servicio de cualquier índole, pueden influir factores como el entorno económico en el que se desarrollan e interactúan las personas, el estrato social que frecuentan, el nivel educativo y la influencia de padres o amigos, entre otros. Estos factores se convierten en un punto de referencia para estudiar la adopción y el uso de servicios innovadores (Ponce y Calderón, 2016)

Principalmente las investigaciones han basado sus enfoques teóricos en la conducta de las personas, utilizando la Teoría de la Acción Planificada de Fishbein y Ajzen (1975-1980), mostrando elementos de la conducta de las personas que inciden directamente en la toma de decisiones por adoptar una innovación. De igual manera, los estudios han basado sus enfoques teóricos en la Teoría de la Difusión de Innovaciones (Rogers, 2003), que básicamente orienta sus argumentos en aquellos elementos requeridos para dar a conocer ante los individuos, todos aquellos aspectos fundamentales que presenta un proceso de innovación. 


\section{REVISIÓN DE LA LITERATURA}

Se realiza una revisión del estado del arte con el fin de indagar sobre las teorías que han sido más utilizadas por los investigadores para llevar a cabo los procesos de adopción de las personas ante distintos elementos de innovación. Es necesario mencionar que se ha llevado a cabo una exhaustiva búsqueda de información relacionada con palabras claves tales como: Adopción de la innovación, innovación financiera, toma de decisiones, factores socioculturales, factores demográficos, entre otras. Estas palabras claves han permitido generar una estrategia de análisis bibliométrico utilizando bases de datos que contienen publicaciones académicas de gran calidad, como es el caso de Scopus, Web of Science, SciELO, DOAJ, Journal Citation Reports, EBSCO, entre otras, sin embargo, no se ha logrado encontrar publicaciones relacionadas con los factores que inciden en la adopción de servicios financieros innovadores.

\section{TEORÍA DE LA DIFUSIÓN DE LAS INNOVACIONES}

Existen varias teorías que sustentan que la conducta de las personas se ve influenciada por sus valores socioculturales y demográficos incidiendo en su voluntad de adoptar nuevos modelos innovadores de servicios. La Teoría de la Difusión de las Innovaciones, nos ayuda a entender la adaptación a una nueva innovación. En otras palabras, esta teoría ayuda a explicar el proceso de cambio social. La novedad de la idea percibida por el individuo determina su reacción ante ella (Rogers, 2003).

Everett Rogers (1962), sociólogo norteamericano, desarrolló como parte de su Tesis Doctoral en Sociología la Teoría de la Difusión de la Innovación (TDI). Esta teoría se basa en la identificación de cinco categorías interrelacionadas y mutuamente excluyentes. La selección de estas características "se realizó basada en escritos anteriores (de Rogers) e investigaciones, y en el deseo de la máxima generalización" (Rogers, 1962, p. 124).

Rogers plantea un modelo teórico basado en cuatro elementos: La innovación, los canales de comunicación, el tiempo y el sistema social. Estas variables son identificables en toda investigación sobre difusión y un proceso de decisión de la innovación dividido en varias etapas, que el individuo o la organización han de superar para alcanzar el definitivo grado de adopción de una innovación.

Esta teoría establece que el generar una innovación requiere de un proceso, la etapa inicial de este es reconocer un problema o necesidad, 
el cual impulsa la investigación y la realización de actividades para otorgar la solución.

La etapa siguiente es la ejecución de la investigación básica aplicada al problema o necesidad identificada anteriormente, puesto que la gran parte de las innovaciones surgen de actividades de investigación científica y su interacción con la práctica, generando tecnologías que se entienden como diseño de tareas prácticas que reducen la incertidumbre entre la relación causa-efecto en acciones que buscan alcanzar un determinado resultado (Rogers, 1983).

Cuando se encuentre una posible solución al problema o necesidad se comienza con la etapa tres, llamada desarrollo y consiste en adecuar la idea o producto que satisfaga las necesidades de una audiencia potencial. Consecuentemente, una vez que se ha corroborado que la solución sí otorga la respuesta al problema, continúa la fase de comercialización; se lleva a cabo actividades de producción, mercadeo, distribución, entre otros.

La última fase es la de difusión y adopción, esta fase formula las unidades adoptantes para la difusión.

El autor señala que si el problema es prioritario existe más presión para adoptar la innovación y esto provoca la rápida difusión. La última etapa del proceso es la llamada "consecuencias" porque verifica si la innovación consiguió realmente satisfacer la necesidad o problema y a la vez identifica si se crearon nuevos problemas con base en la innovación. Es importante resaltar que la secuencia de estas etapas no es estrictamente en el orden mencionado y tampoco obedece a un comportamiento lineal (Rogers, 1983).

Esta teoría también considera el proceso de decisión para adoptar una innovación, el cual consiste en la recolección y procesamiento de información con el propósito de reducir la incertidumbre respecto de las ventajas y desventajas de una innovación. El proceso consta de cinco etapas que se detallan a continuación.

Conocimiento: El proceso de decisión inicia con el conocimiento sobre la innovación. La unidad adoptante se enfoca en aprender cómo funciona la nueva idea u objeto.

Persuasión: Una vez que tiene el conocimiento necesario se inicia la etapa de persuasión, la cual consiste en formarse una actitud sobre tal 
innovación, ya sea favorable o desfavorable, con base en el conocimiento adquirido, un alto componente afectivo y sus proyecciones sobre adoptar o no la innovación, para lo que busca opiniones de otros, entre ellos expertos, o bien personas similares, cuyas opiniones son significativamente influyentes en la decisión de adoptar algo nuevo. La actitud que se forme la unidad adoptante va a dar pie a la decisión de adoptar o rechazar la innovación, aunque no siempre se da en el mismo sentido.

Decisión: En la tercera etapa la de decisión, el individuo ejecuta actividades encaminadas a definir una posición sobre la innovación, entre las que se encuentran pruebas previas, cuando sea posible, para medir el desempeño de la innovación.

Implementación: Una vez que la unidad adoptante ha aceptado la innovación, es el momento de implantarla. En las etapas anteriores el ejercicio es a nivel mental, es aquí donde se requiere un cambio de comportamiento para poner en práctica la nueva idea. Se cree que esta etapa finaliza cuando la idea considerada como nueva pierde sus cualidades distintivas.

Confirmación: El proceso concluye con la etapa de confirmación en la cual, el adoptante busca información para asegurarse de que la decisión que se ha tomado, de adoptar o rechazar, es la correcta y así reducir el desequilibrio interno o incomodidad, pues incluso puede cambiar de parecer después de haber decidido adoptar la innovación (Rogers, 1983).

Figura 1. Teoría de la Difusión de las Innovaciones.

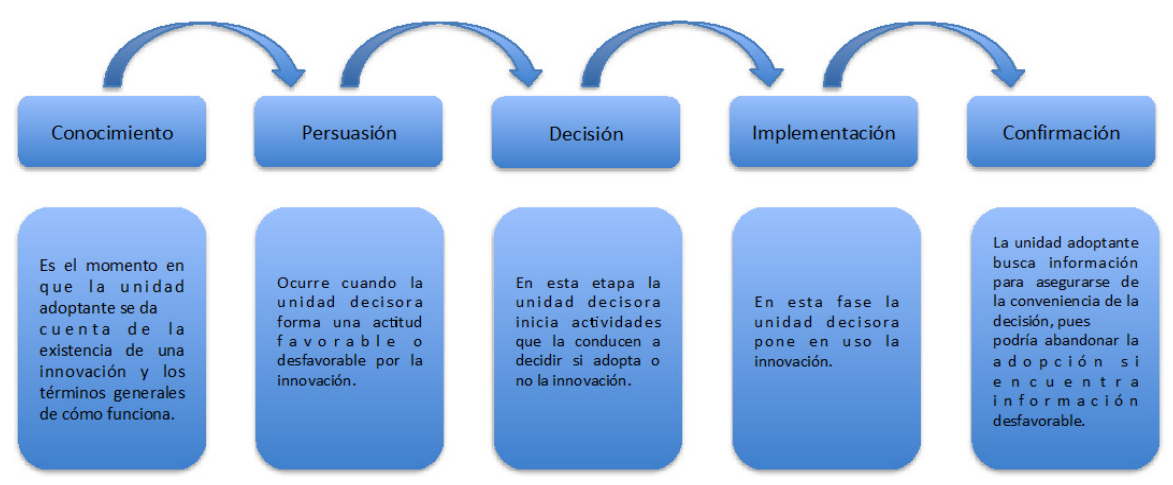

Fuente: Teoría de la Difusión de las Innovaciones. Rogers (1983). 
La teoría de la difusión de la innovación expone que una innovación posee cinco características básicas, las cuales provocan que su proceso de adopción sea más fácil, pues causan una actitud más favorable. Rogers (1983) define estos atributos de la siguiente manera:

a) Ventaja relativa: Grado en que una innovación es percibida como mejor que la idea a la que remplaza.

b) Compatibilidad: Grado en que una innovación es percibida como consistente con los valores existentes, las experiencias pasadas, las necesidades y los adoptantes potenciales.

c) Complejidad: Grado en que una innovación es percibida como relativamente difícil de entender y usar.

d) Experimentación: Grado en que una innovación puede ser experimentada sobre una base limitada.

e) Tangibilidad: Grado en que los resultados de una innovación son visibles para otros.

Como elemento adicional, Rogers (2003), plantea que la adopción de una innovación es un proceso de distintas etapas; proceso que es influenciado por antecedentes propios del entorno en el que se va a implementar la innovación tales como, la identidad del actor o la percepción de la situación, lo que produce un resultado que, a manera de resumen, se manifiesta en la decisión de adoptar o rechazar una innovación. Este proceso se divide en cinco etapas, en las cuales se ponen de manifiesto las cinco características perceptibles de la innovación.

Estas etapas inician con la búsqueda de información por parte de la unidad de decisión, la cual, busca disminuir el nivel de incertidumbre asociado a la aparición de una innovación. Posteriormente la entidad generará una percepción sobre la innovación misma, generando una decisión ya sea de adopción o rechazo de la solución novedosa.

Luego de implementar la decisión tomada se confirmará la misma mediante la comparación de los resultados obtenidos con las percepciones desarrolladas en etapas anteriores. El diseño del proceso de decisión de innovaciones se muestra a continuación. 
Figura 2. Diseño esquemático del proceso de decisión de innovaciones.

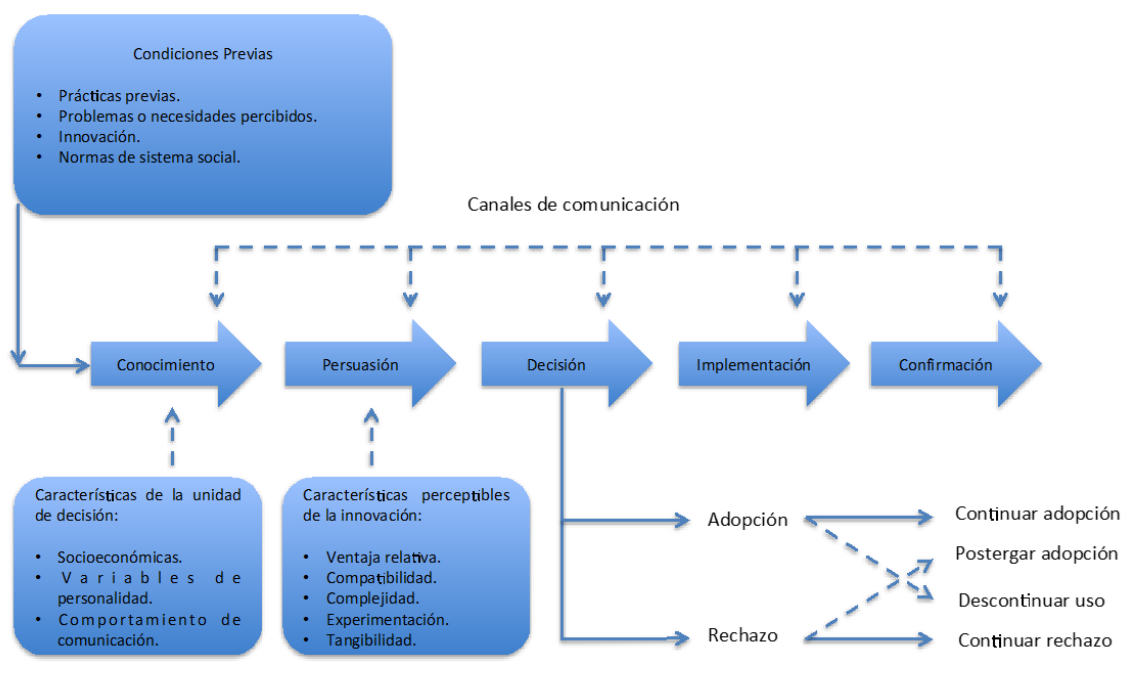

Fuente: Rogers, E. M. (2003). Diffusion of innovations. New York: The Free Press.

Además, el autor realiza un aporte revelador de la Teoría de la Difusión de las Innovaciones, pues plantea una serie de categorías de las personas u organizaciones que adoptan innovaciones, sumado a la cuantificación de la participación de dichos perfiles en un proceso de adopción e implementación de una innovación en un sector específico. 
Tabla 1. Características de los perfiles frente a la innovación.

\begin{tabular}{|c|l|}
\hline Perfil & \multicolumn{1}{|c|}{ Características } \\
\hline \multirow{4}{*}{ Innovadores } & Orientación al riesgo. \\
\cline { 2 - 3 } & Buenos niveles de recursos financieros. \\
\cline { 2 - 2 } Primeros & Habilidad para entender y aplicar tecnologías complejas. \\
\hline \multirow{2}{*}{$\begin{array}{c}\text { Mayoría } \\
\text { Temprana }\end{array}$} & Alto nivel de liderazgo de opinión. \\
\cline { 2 - 2 } & Fijadores de tendencias. \\
\cline { 2 - 2 } & Tiempo de decisión más alto. \\
\hline \multirow{4}{*}{ Mayoría Tardía } & Adopción por presiones económicas o sociales. \\
\cline { 2 - 3 } & Recursos realizadas con escepticismo. \\
\cline { 2 - 2 } & Rescasos para la adopción. \\
\hline \multirow{3}{*}{ Relegados } & Sin liderazgo de opinión. \\
\cline { 2 - 2 } & Resistencia a la innovación. \\
\hline & \\
\hline
\end{tabular}

Fuente: Rogers, E. M. (2003). Diffusion of innovations. New York: The Free Press.

Los canales de comunicación, el tiempo para comunicar la innovación, la estructura de comunicación, concentración del poder y existencia de líderes de opinión o agentes de cambio dentro del sistema social también son variables que afectan la adopción, según esta misma teoría (Rogers, 1983). 


\section{EL ESTADO DE LA TEORÍA DE LA DIFUSIÓN DE LAS INNOVACIONES EN LA ACTUALIDAD}

La teoría no muestra cambios tan radicales, y más bien son muchos los aportes que la complementan, por ejemplo Liang, Hou, Chen y Hu (2020), utilizan la Teoría de la Difusión de la Innovación para explicar cómo, por qué y a qué velocidad se extiende una nueva idea, práctica u objeto a lo largo del tiempo entre los miembros de un sistema social.

También, Barrett, Pas y Lindstrom (2020), han utilizado la Teoría de la Difusión de las Innovaciones para hacer estudios en varios campos, por lo que las innovaciones se refieren a cualquier práctica o herramienta percibida como novedosa por el adoptante, que puede ser una organización o un individuo (Rogers, 2003). La adopción de la innovación es altamente idiosincrática y se ve afectada por procesos organizacionales, cultura y apertura al cambio, estilo de liderazgo, recursos, infraestructura y relaciones sociales (Greenhalgh et al., 2004).

Otros autores como Yoon, Lim, y Park (2020), utilizaron la Teoría de la Difusión de Innovaciones desarrollada por Rogers (2003) para explicar procesos de adopción de la innovación. En la investigación de sistemas de información, las características de innovación también se han utilizado ampliamente en estudios relacionados con la adopción de tecnología de la información en las organizaciones (Oliveira y Martins, 2011), así como su aceptación por parte de los individuos (Venkatesh et al., 2003 ). En tales estudios, las características de innovación se han aplicado con frecuencia en combinación entre la Teoría de la Adopción Tecnológica y la Teoría de la Difusión de las Innovaciones (p. Ej., Oliveira, Thomas y Espadanal, 2014; Zhu, Kraemer y Xu, 2003)

Por lo tanto y dado que los objetivos de esta investigación están enfocados en conocer factores socioculturales y demográficos de las personas, para llevar a cabo un proceso de adopción de la innovación financiera, se considera oportuno utilizar la Teoría de la Difusión de las Innovaciones de Everett Rogers como una de las teorías de referencia de esta investigación. 


\section{TEORÍA DE LA CONDUCTA PLANIFICADA}

La Teoría de la Conducta Planificada se ha utilizado para explicar el comportamiento de individuos en numerosos dominios, como por ejemplo, la industria de alimentos orgánicos (Yazdanpanah y Forouzani, 2015), comportamiento de compra verde (Wang et al., 2016; Yadav y Pathak, 2016), vivienda sostenible (Judge et al., 2019), sociedades sostenibles (Kaffashi y Shamsudin, 2019), reducción de la contaminación (Cordano y Frieze, 2000 ), industria de la construcción (Teo y Loosemore, 2001; Mak et al., 2019), entre otras. Investigaciones anteriores sugieren que el comportamiento humano es complejo y no puede ser capturado por un modelo uniforme.

Aunque la Teoría de la Conducta Planificada explica bien los patrones de comportamiento fundamentales, debe personalizarse para un propósito específico para una mayor precisión y confiabilidad. Una mejor comprensión del comportamiento humano y los factores que lo afectan pueden ayudar al planteamiento de la toma de decisiones para llevar a cabo la adopción de la innovación.

Estos autores han estudiado el comportamiento del consumidor aplicando la Teoría de la Conducta Planificada, y han demostrado la capacidad del modelo para predecir el comportamiento con respecto a ciertos productos como alimentos procesados (Dunn, Mohr, Wilson y Wittert, 2011; Mahon, Cowan y McCarthy, 2006 ; Olsen, Sijtsema, \& Hall, 2010; Ricci, Banterle, \& Stranieri, 2018; Saba et al., 2008; Stranieri, Ricci, \& Banterle, 2017).

Otros estudios han utilizado la Teoría de la Conducta Planificada para investigar la orientación de conveniencia en lo que respecta a los estilos de vida, señalando que la presión del tiempo es un impulsor de la demanda de conveniencia (Brunner et al., 2010; Candel, 2001; De Boer, McCarthy, Cowan y Ryan, 2004).

El modelo de comportamiento planificado se utiliza con una amplia gama de propósitos sobre el comportamiento humano y tiene un papel importante en la predicción de factores sociopsicológicos relacionados con las intenciones (Chen, 2017). Además, la teoría identifica tres clases de antecedentes de la intención de asumir (o no asumir) un comportamiento. Primero, la actitud hacia el comportamiento representa la valoración positiva o negativa del individuo con respecto a sus propias creencias y valores subjetivos. La actitud indica la intención positiva y negativa de una persona para realizar un comportamiento específico, y 
si una persona tiene una actitud positiva hacia un comportamiento, es más probable que lo haga (Ajzen, 1985, 1991; Davis et al., 2002; Lapple y Kelley, 2013). Por ejemplo, varios estudios han resaltado la importancia de la actitud en la predicción de comportamientos ecológicos en diversos campos, como el ahorro de energía en el hogar, la compra de productos ecológicos y la compra de vehículos ecológicos (Webb et al., 2013; Wang et al., 2016; Yadav y Pathak, 2016).

Segundo, la norma subjetiva refleja la "presión social" relacionada con asumir (o no asumir) el comportamiento. En tercer lugar, el control conductual percibido representa los factores percibidos como facilitadores o barreras con respecto al comportamiento. Además de ser un antecedente de intención, el control conductual percibido también se puede usar como un proxy del control real sobre el comportamiento, asumiendo un vínculo directo con el logro del comportamiento (Russell y Fielding, 2010). No obstante, diferentes investigadores desarrollaron el comportamiento planificado según el sujeto y el público objetivo (Azadi et al., 2019).

Ajzen, como uno de losfundadores deesta teoría, afirma que podrían considerarse nuevos componentes y estructuras de comunicación para mejorar esta teoría (Ajzen, 2005). Por lo tanto, muchos investigadores han estudiado otras variables que no están en este marco y creen que aumentar otras variables puede mejorar la capacidad de predicción del modelo (Yadav y Pathak, 2016; Bird et al., 2018).

La Teoría de la Conducta Planificada a menudo se usa para examinar las motivaciones a nivel individual para participar en comportamientos voluntarios (Scott et al., 2019). En particular, la Teoría de la Conducta Planificada se utiliza principalmente para predecir la intención de adoptar un comportamiento. Además, se ha demostrado que es útil para comprender los procesos de cambio de comportamiento, con el objetivo de desarrollar intervenciones de cambio de comportamiento basadas en la comunicación, la persuasión y la información (Hardeman et al., 2002).

Ajzen (1985) propone que el determinante inmediato de una conducta es la intención, sin embargo como la intención puede cambiar, también ha propuesto dos predictores para determinar la intensidad de la intención. Según el autor la intención por ejecutar una determinada conducta, está en función de cuán favorable sea la actitud de la persona por el comportamiento, así como por la medida en que crea que otras personas piensan que debería hacerlo; sin obviar los factores fuera de control del individuo que afectan tanto la intención como la conducta. Estas interacciones se detallan gráficamente en la figura siguiente. 
Figura 3. Teoría de la Conducta Planificada de Ajzen.

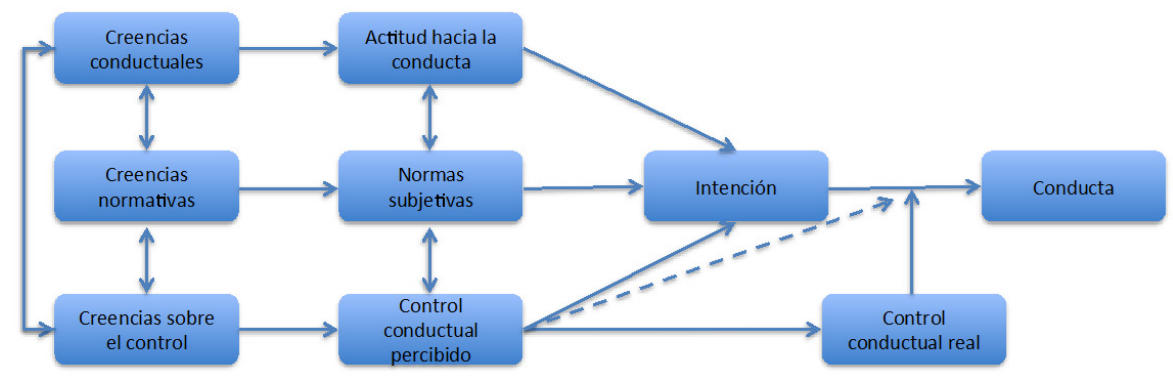

Fuente: Teoría de la conducta planificada. Ajzen (2006).

La Teoría de la Conducta Planificada propone que la actitud hacia el comportamiento, se forma a partir de una combinación o ponderación entre la actitud por intentar ejecutar el comportamiento de manera exitosa, e intentarlo fracasadamente. La actitud predominante depende de las creencias del individuo sobre la presencia o ausencia de los factores personales o externos sobre los cuales no tiene control. Estas actitudes son determinadas por las creencias internas del individuo, las cuales a su vez están relacionadas con las consecuencias de la ejecución del comportamiento que tiene éxito al alcanzar el objetivo (Ajzen, 1985)

Además, la Teoría de la Conducta Planificada de Fishbein y Ajzen (1975-1980) menciona que nuestras creencias determinan nuestras actitudes y nuestras normas subjetivas y por ello, aunque de forma indirecta, determinan nuestras intenciones y conductas.

Ajzen (1985) establece que las intenciones son en un sentido estricto sólo una intención de una persona por intentar ejecutar un comportamiento, pero no asegura que en realidad realice ese comportamiento. Si la intención no logra predecir el comportamiento, sería porque la intención inicial cambió; sin embargo, si la intención sí logra predecir el comportamiento de la persona, pero no el logro del objetivo que se pretende con tal conducta sería porque existen factores fuera del control del individuo que le impiden lograr el objetivo, aunque haya mantenido la intención de ejecutar ese comportamiento. Los factores que influyen pueden ser internos como las diferencias individuales, de información, de habilidades y destrezas, de fuerza de voluntad o de emociones; pero también pueden ser factores externos como el tiempo y las circunstancias adecuadas (oportunidad) en que se pretende ejecutar un determinado comportamiento, así como la 
dependencia de otras personas para lograr el objetivo de la conducta.

Esta relación se da principalmente cuando la conducta es voluntaria; pero cuando es obligatoria o impuesta, las creencias están asociadas a las consecuencias de una conducta que fracasa en el logro del objetivo. De igual manera, el análisis anterior según el autor de la teoría, se puede aplicar a las normas subjetivas, no obstante la distinción es menos relevante porque cuando el individuo cree que la conducta es la deseada por otras personas que considera importantes, no piensa tanto en el éxito o fracaso, sino en la deseabilidad social y por ende, toma la aceptación de los demás como una aprobación de que logrará el objetivo que persigue con ese comportamiento (Ajzen, 1985).

El control de la conducta real consiste en las habilidades, fuerza de voluntad, factores físicos requeridos y la intención del comportamiento, estos, unidos con condiciones favorables o desfavorables que llegue a percibir la persona, van a predecir la probabilidad del éxito de que se ejecute el comportamiento, el mismo autor sobresalta que por el hecho de que el control de la conducta es una percepción y no es exento de ser subjetivo, puede provocar el detrimento de medir con precisión el control del comportamiento que se posee (Ajzen, 1985).

Por lo anterior, los autores mencionan que el uso de un servicio innovador puede estar condicionado por la intención de uso, misma que se ve afectada por las creencias de las personas, y por otro lado, por los beneficios percibidos, debido a que cuando las personas consideran que su desempeño se va a mejorar en alguna medida, se ven más motivados por adoptar un servicio innovador, pues los beneficios que normalmente trae una mejora en el desempeño son atractivos para el adoptante (Davis, 1989).

Tomando en cuenta que la utilidad es la característica más determinante en la formación de la intención de uso, pues fue empíricamente comprobada que tiene la capacidad para predecir el uso de computadoras por las personas, Davis et al. (1989) concluyen que las personas podrían estar dispuestas a tolerar un servicio innovador difícil de utilizar, siempre que este sea funcional, pero ningún nivel de facilidad de uso compensará la ausencia de utilidad (Davis et al. 1989). 


\section{EL ESTADO DE LA TEORÍA DE LA CONDUCTA PLANIFICADA EN LA ACTUALIDAD}

Heinze y Heinze (2020), indican que la investigación sobre la adopción de la innovación individual se basa en perspectivas, como los modelos de aceptación tecnológica (Venkatesh y Morris, 2000) y la Teoría de la Conducta Planificada (Ajzen 1991), para considerar la influencia de las características individuales (por ejemplo, experiencia y actitudes) (Bekkering y Hutchison 2009), características de innovación, por ejemplo, la utilidad percibida y facilidad de uso percibida.

De igual manera, Sourabh, Shaleen, Nikunj y Kalyan (2020) indican que la Teoría de la Conducta Planificada (Ajzen, 1985, 1991) ha sido la teoría más ampliamente utilizada para predecir el comportamiento proambiental a nivel individual. Los investigadores han ampliado la Teoría de la Conducta Planificada al agregar más variables para mejorar la precisión de la predicción. Las variables adicionales incluyen normas morales (Yazdanpanah y Forouzani, 2015; Kaffashi y Shamsudin, 2019), la falta percibida de instalaciones (Stoeva y Alriksson, 2017), identidad del consumidor (Yazdanpanah y Forouzani, 2015; Judge et al., 2019), instituciones y gobernanza (Kaffashi y Shamsudin, 2019; Mak et al., 2019), preocupaciones ambientales (Kaffashi y Shamsudin, 2019) o conciencia y avances tecnológicos (Kaffashi y Shamsudin, 2019).

Así mismo, Contini, Boncinelli, Marone, Scozzafava y Casini (2020), utilizaron como marco teórico de referencia la Teoría de la Conducta Planificada (Ajzen, 1991), que se ha utilizado ampliamente para predecir y explicar el comportamiento en diferentes ámbitos.

Savari y Gharechaee (2020), se han propuesto varias teorías y modelos para estudiar las intenciones de comportamiento y la voluntad de que la Teoría de la Conducta Planificada de Ajzen (Ajzen, 1991) sea una de las teorías más utilizadas. La Teoría de la Conducta Planificada al considerar la interacción entre factores individuales, sociales y ambientales, es una herramienta valiosa y poderosa para predecir la intención e intenciones de comportamiento (Rahmaninkoshkaki y Zarei, 2018). 
Perri, Giglio y Corvello (2020), utilizaron la Teoría de la Conducta Planificada para estudiar variables que afectan las actitudes de los consumidores hacia la adopción de comportamientos inteligentes en el uso de energía, particularmente su actitud hacia la adopción de patrones de consumo impulsados externamente asociados con el uso de redes inteligentes (Faiers et al., 2007).

Por estas razones antes descritas, en este estudio también se adopta la Teoría de la Conducta Planificada (Ajzen, 1985; 1991; 2012) como marco teórico, pues el objetivo se centra en el análisis de factores socioculturales y demográficos que inciden en la conduca del individuo, para llevar a cabo el proceso de toma de decisiones para adoptar un servicio financiero innovador. 


\section{FUNDAMENTOS TEÓRICOS Y EMPÍRICOS PARA UN POSIBLE MODELO DE INVESTIGACIÓN}

Es importante mencionar que cuando se habla del término "aceptación" se refiere al hecho de que el individuo no se opone a la existencia de un nuevo producto o servicio financiero, mientras que cuando se refiere al término "adopción", se hace referencia al hecho de que el individuo utiliza el servicio financiero como parte de sus recursos para interactuar en el mercado financiero.

A todo esto, el riesgo se convierte en un factor importante de analizar, y aquí se refiere a la probabilidad estadística de un evento adverso. Que tiene una causa conocida o hipotetizada. Aplicando esta perspectiva técnica, el análisis de los científicos sobre el riesgo permite la comparación de riesgos muy diferentes. Un riesgo de baja probabilidad, que tiene graves consecuencias.

Los estudios dentro de la psicología han demostrado que las evaluaciones de riesgo no se basan únicamente en un cálculo del producto de la probabilidad estadística estimada y del efecto estimado. El modelo psicométrico de percepción de riesgo involucra dimensiones tales como un conocimiento, grado de novedad y familiaridad, grado de control personal y catastrófico potencial (Slovic, 2000) citado en (Boholm, 2003).

Las decisiones sobre el riesgo y la gestión del riesgo están socialmente integradas, conformado por nociones basadas en la cultura sobre el estado del mundo, en lo que el mundo consiste y cómo funciona. Las nociones culturales nos dicen de manera intuitiva lo que es potencialmente peligroso y dañino y, lo que no lo es, nos proporciona modelos explicativos que nos digan por qué las cosas se comportan como lo hacen, y pautas morales con respecto a por qué las cosas o acciones son buenas o correctas, mientras que otras son malas o incorrectas (Douglas, 1992) citado en Boholm (2003).

De igual manera, es importante tomar en cuenta aspectos relacionados con la percepción al riesgo que tienen las personas, pues existe un triángulo de conexiones entre incertidumbre, riesgo y creatividad; de hecho, una tolerancia saludable al riesgo y a la incertidumbre pueden estimular el pensamiento creativo (Anderson, 2011), y fomentar el emprendedurismo y el uso de nuevos e innovadores servicios financieros, pero de igual manera pueden incidir negativamente en su aceptación y adopción. De esta información se deriva la hipótesis siguiente:

H1. Las personas que poseen una mayor aversión al riesgo utilizan menos los servicios financieros nuevos e innovadores. 
En efecto, Druckman y McDermott (2008) muestran cómo la propensión al riesgo puede fluctuar a lo largo del tiempo. En particular, las emociones pueden alterar la propensión al riesgo en forma sistemática y predecible (Bower, 1981; Johnson y Tversky, 1983; DeSteno, Petty, Rucker y Wegener, 2000; Lerner, González, Small y Fischhoff, 2003; Mackuen, Wolak, Keele y Marcus, 2005; Druckman y McDermott, 2008). Así, por ejemplo, Druckman y McDermott (2008) sostienen que algunos estados emocionales, como el estrés o la ansiedad y la ausencia de entusiasmo, pueden incrementar la aversión al riesgo. En otras palabras, estos estados emocionales podrían afectar el grado de aversión al riesgo y, por consiguiente, alterar su efecto en la decisión (Ponce, Mena-Mora y Rottinghaus, 2012).

No obstante a esto, es importante fomentar la cultura de innovación, pues con ella se crea valor y fomenta la expectativa de crecimiento, pues la cultura de la innovación del conocimiento se entiende como una clase de valor, comportamiento y sistema institucional que consigue ventajas competitivas.

Dado que la innovación es clave en la supervivencia de las empresas ya que, para competir en los actuales entornos cada vez más cambiantes, éstas han de crear nuevos productos y servicios (Cefis y Marsili, 2006), se hace necesario fomentar el desarrollo de nuevas ideas, y para esto es importante que las personas cuenten con cierta educación académica formal, pues el nivel educativo constituye un recurso fundamental para el desarrollo de nuevas ideas y para alcanzar el éxito en las iniciativas innovadoras que persigue la empresa (Van der Vegt y Janssen, 2003). Las actividades de innovación requieren creatividad y conocimientos técnicos y especializados, y son los empleados con alto nivel formativo los que los tienen (Hayton y Kelley, 2006). También es necesario mencionar que la baja escolaridad es un indicador de pobreza que obstaculiza la adopción (Marín et al. 2006, citado por Zepeda, Velasco, Nahed, Hernández y Martínez (2016).

A mayor nivel de educación, mayores son las oportunidades de aportar innovación en diversos aspectos para impulsar el crecimiento económico. Los países con un pequeño stock de capital humano "educación escolar" pueden tener una tasa de crecimiento más lenta que los países desarrollados con un gran stock de capital humano "educación terciaria" (Salam, Hafeez, Mahmood, lqbal y Akbar, 2019). Por lo cual se establece la siguiente hipótesis. 
H2. Las personas son más propensas a tomar la decisión de aceptar y adoptar servicios financieros innovadores según su grado de educación académica sea mayor.

Pero la innovación por sí misma no implica el éxito en la gestión de una empresa o institución, también la toma de decisiones pone en juego numerosos procesos cognitivos, entre ellos el procesamiento de los estímulos presentes en la tarea, el recuerdo de experiencias anteriores y la estimación de las posibles consecuencias de las diferentes opciones (Barrutieta, 2011). Las emociones guían la toma de decisiones, simplificando y acelerando el proceso, reduciendo la complejidad de la decisión y atenuando el posible conflicto entre opciones similares (Bechara, Damasio y Damasio, 2000), por lo que la aceptación y sobre todo la adopción del uso de un servicio financiero innovador está sujeta a la toma de decisiones de los demandantes, quienes como se ha mencionado, están afectados por diversos factores internos y externos en sus procesos de toma de decisión, por ejemplo, Rodríguez y Acevedo (2002) identificaron la relación que existe entre la cultura y los procesos de toma de decisiones. Sin embargo, la mayoría de las decisiones son tomadas en un grupo, especialmente la familia (Cherfi, 2011), pues existe un vínculo entre los valores y el modo de consumo (Jolibert y Valette-Florence, 1985).

Así mismo, comparaciones entre países se pueden hacer utilizando las etapas de los autores. Por ejemplo, un país puede estar en la etapa de prueba de adopción de tecnología o cualquier otro elemento de innovación, mientras que un segundo país acaba de entrar en la etapa de interés. Como resultado, caracterizaríamos que el primer país está más lejos en la adopción de tecnología que el segundo país (Weber y Kauffman, 2011).

En la adopción, influyen factores como el entorno en el que se desarrollan las personas, el estrato social, el nivel educativo y la influencia de padres o amigos. Estos factores se convierten en un punto de referencia para estudiar la adopción y el uso de servicios innovadores (Ponce y Calderón, 2016). Así entonces:

H3. Elementos culturales de la familia, inciden directamente en la toma de decisiones de las personas para adoptar servicios financieros innovadores. 
Por otra parte, Park, Lee y Cheong, (2007) señalan que la motivación es un factor que influye en el uso y la adopción de la innovación. El disfrute percibido en el uso de la innovación, definido éste como el grado en que la actividad de usar un producto o servicio innovador se percibe como personalmente agradable por sí misma, aparte del valor instrumental que éste genera. Roca y Gagné (2008) indican que el disfrute percibido afecta directamente tanto a la utilidad percibida como a la facilidad de uso percibida, lo que puede contribuir a la adopción de un servicio financiero innovador.

El constructo utilidad percibida está basado en los estudios sobre las motivaciones, las expectativas y las investigaciones de los sistemas de información realizados, entre otros autores, por Vroom (1964), Larcker y Lessig (1980) y Bandura (1982) citado en López y López (2011).

Las construcciones clave en el proceso de decisión de innovación son los atributos percibidos de la innovación. Cada persona evaluará la innovación basándose en cinco atributos:Ventaja relativa, compatibilidad, complejidad, capacidad de control y observabilidad (Sereenonchai, Xu, Arunrat y Yu, 2017).

Chen, Chen, Lin y Yeh (2007) muestran que el disfrute percibido afecta significativamente la percepción sobre la utilidad y Zhang, Zhao y Tan (2008) señalan que el disfrute percibido tiene un efecto positivo sobre la intención del comportamiento de las personas por la utilización de un producto o servicio innovador.

Aunado a esto, existen elementos que pueden incidir en la toma de decisiones de las personas por adoptar la innovación, es así como Salas, Sagarnaga y Zavala (2013) citado por Zepeda, Velasco, Nahed, Hernández y Martínez (2016) menciona que las personas que ya están próximas a ser adultos mayores tienen menor tendencia a adoptar procesos de innovación, pues en su estudio, las personas de edad avanzada fueron los que menos adoptaron nuevas tecnologías debido a su innovación. Por lo anterior, es de consideración que la edad es un factor importante en los procesos de adopción de la innovación, por lo que se establece la siguiente hipótesis.

H4. Las personas con más edad, tienen menor tendencia a utilizar servicios financieros nuevos e innovadores. 
De igual manera, las personas que poseen mayor experiencia en el uso de servicios financieros, o que han sido influenciados por un mayor nivel de capacitación en este tipo de servicios financieros, es posible que puedan mostrar un mayor nivel de influencia positiva hacia el uso de los mismos y por ende a ejercer un proceso de adopción de servicios financieros innovadores con mayor rapidez, dado que la capacitación influye positivamente en la adopción de nuevas tecnologías y procesos innovadores (Forero, Rojas y Arguelles, 2013), por lo que la adopción de nuevos servicios financieros innovadores puede ser incrementada por programas de capacitación previos a la salida al mercado de este tipo de servicios innovadores.

Otras explicaciones alternativas sugieren que la adopción puede ser influenciada por subsidios, incluyendo incentivos de empresa a consumidor o de empresa a empresa (Riggins et al. 1994), citado en Weber y Kauffman (2011).

Las organizaciones deben proporcionar condiciones de facilitación, que incluyen la extensión y el tipo de apoyo proporcionado a las personas que influirían en su uso de la innovación. Se cree que las condiciones de facilitación incluyen la disponibilidad de capacitación y la provisión de apoyo. Se ha identificado que las condiciones de facilitación tienen un efecto sobre la infusión o la adopción de una serie de innovaciones. Estos factores incluyen la capacitación apoyo gerencial e incentivos. Estas influencias afectan la conciencia de una persona sobre el funcionamiento y la aplicación de una innovación, su utilidad y ajuste con el trabajo, lo que lleva a su adopción (Frambach y Schillewaert, 2002) citado en (Talukder, 2014). Por esta razón se establece la hipótesis siguiente:

H5. Personas con mayor experiencia o capacitación en el uso de servicios financieros, poseen mayor nivel de aceptación y adopción de servicios financieros innovadores.

Esimportante mencionarquelos procesos deinnovación conllevan elementos de riesgo que son más fáciles de asumir dependiendo de las consecuencias que afronten las personas por tomar la decisión de adoptar la innovación, es así, donde los procesos de innovación se ven afectados directamente por factores externos a su propia naturaleza, de esta manera las personas que tienen mayores recursos económicos pueden asumir mayor riesgo, pues las consecuencias pueden ser absorbidas de manera más expedita, minimizando sus efectos, no obstante personas con menores ingresos tienen una menor oportunidad 
de innovar, debido a sus limitaciones económicas, pues los estudios han revelado que las personas no cambian de un sistema tradicional, más seguro y experimentado, a uno nuevo que pudiera estar asociado a riesgos económicos (Aldy, Hrubovcak y Vasavada, 1998).

Sereenonchai et al. (2017) se centra principalmente, en las decisiones de adopción de las personas, y varios factores que afectan sus decisiones. Las construcciones clave en el proceso de decisión de innovación son las características socioeconómicas del individuo. Así entonces, la hipótesis número seis, se describe de esta manera:

H6. Las personas con niveles económicos menores tienen menos tendencia a utilizar servicios financieros nuevos e innovadores.

El género se considera un elemento vital para explicar las diferencias e identidades en la sociedad moderna (Walby, 1990), y en el caso de la adopción de la innovación, la literatura indica al género como fundamental para entender diferencias en el rol de las percepciones de utilidad y facilidad de uso como determinantes de esta adopción (Ong y Lai, 2006; Sánchez- Franco, Villarejo-Ramos y Rondan-Cataluña, 2006; Venkatesh y Morris, 2000).

De acuerdo a esto, Kim y Forsythe (2008) indican que no existen diferencias estadísticamente significativas entre hombres y mujeres en el proceso de adopción de un particular producto o servicio innovador. No obstante a esto, existen evidencias en otros estudios, sobre diferencias asociadas al género en la adopción de procesos de innovación.

Ong y Lai (2006) muestran que las percepciones de los hombres acerca de la utilidad percibida, la facilidad de uso percibida y la intención de comportamiento para el uso de productos innovadores son más altas que las percepciones de las mujeres. Además, la utilidad percibida influye en la intención de comportamiento para el uso de productos y servicios innovadores con más fuerza para los hombres que para las mujeres $y$, del mismo modo, la facilidad de uso influye en la utilidad percibida del servicio o producto innovador con más fuerza en las mujeres que en los hombres. 
Por lo anterior, y de acuerdo a la naturaleza de este estudio, y tomando en cuenta que existe limitada información relacionada con el comportamiento de hombres y mujeres ante la adopción de servicios financieros innovadores, se plantea la hipótesis siguiente:

H7. El género en las personas no es un factor determinante en el proceso de la toma de decisión para adoptar servicios financieros innovadores. 


\section{METODOLOGÍA}

\section{DETERMINACIÓN Y SELECCIÓN DE LA MUESTRA}

La población objeto de estudio está representada por una población infinita de individuos, de los cuales se estima que el 95\% están ubicados en el Área Metropolitana, incluyendo zonas como la provincia de Heredia, Alajuela, San José y Cartago, y un 5\% podrían pertenecer a zonas más rurales como Guanacaste, Limón y Puntarenas. De esta población, se estima que el $45 \%$ de los individuos son mujeres y el restante porcentaje son hombres y mantienen una edad promedio que va desde los 18 a los 65 años. Se han considerado como unidades de muestreo las personas de forma directa.

Sin embargo, un paso de especial relevancia en el estudio empírico es el correspondiente a la confección de la muestra sobre la que se va a llevar a cabo la contrastación de las hipótesis. En concreto, en este estudio empírico no hay una escogencia de la muestra, por lo que la muestra utilizada es de tipo probabilístico; debido a que, en caso de ser no probabilística, los sujetos deberían ser seleccionados, dada la conveniente accesibilidad y proximidad de los sujetos para el investigador (Hernández et al., 2010).

Para este caso particular, la encuesta es puesta a disposición de las personas, por medio de una red tecnológica denominada Google Forms que facilita que las personas puedan completar la encuesta en línea, utilizando sus computadoras, incluso sus teléfonos celulares desde cada una de sus ubicaciones.

\section{MEDICIÓN}

Para la adecuada recolección de datos, el proceso consiste en la selección, validación y aplicación del instrumento utilizado para este fin. El cuestionario utilizado es diseñado en escalamiento tipo Likert, basado en la escala desarrollada por O'Reilly (1982), y preguntas abiertas, que se aplica por medio de una encuesta. Dicho cuestionario se elabora después de haber operado las variables y como producto del análisis del objetivo de la investigación. Así se logra redactar un primer cuestionario que es validado por tres profesores con nivel académico de doctorado conocedores del tema y un profesor también con nivel de doctorado, quien es reconocido a nivel internacional como experto en temas de innovación y catedrático de la Universidad de Valencia en España. Así mismo, el cuestionario es revisado por 10 expertos en el 
área de finanzas, con una gran trayectoria y experiencia en puestos gerenciales en importantes instituciones financieras dentro y fuera del país. Así mismo, el instrumento de medición es revisado por un profesional con nivel de maestría en estadística.

Al recibir el instrumento con las observaciones que los validadores hacen, se procede a modificar, organizar y complementar la versión definitiva del cuestionario quedando conformado por 90 ítems en un solo bloque.

El bloque de ítems que van desde el ítem número 1, hasta el ítem número 90, está destinado a la obtención principalmente de datos relacionados con el tema de la influencia de los factores socioculturales, y el bloque final de 6 ítems, pretende obtener información valiosa sobre datos de carácter demográfico sobre el encuestado.

Una vez definidas las variables, es necesario cuantificarlas para poder analizarlas y expresarlas matemáticamente, haciéndose necesario el uso de escalas; es decir, cada una de estas dimensiones ha sido medida a través de un conjunto de ítems ya que no existe un único indicador que refleje la globalidad de cada dimensión. Cada ítem ha sido evaluado sobre una escala tipo Likert, ofreciendo cinco opciones con la siguiente representación: 5= Totalmente de acuerdo, 4= De acuerdo, $3=$ Ni de acuerdo, ni en desacuerdo, 2= En desacuerdo, y $1=$ Totalmente en desacuerdo.

\section{PROCESO DE ELABORACIÓN DEL INSTRUMENTO DE MEDICIÓN}

Luego de la aplicación de los planes piloto, se procede a validar la escala utilizando herramientas estadísticas, con el fin de depurar aún más el cuestionario y proceder a su aplicación con la población. El proceso de creación del instrumento de medición se resume de la siguiente manera: 
Figura 4. Proceso de creación del instrumento de medición.

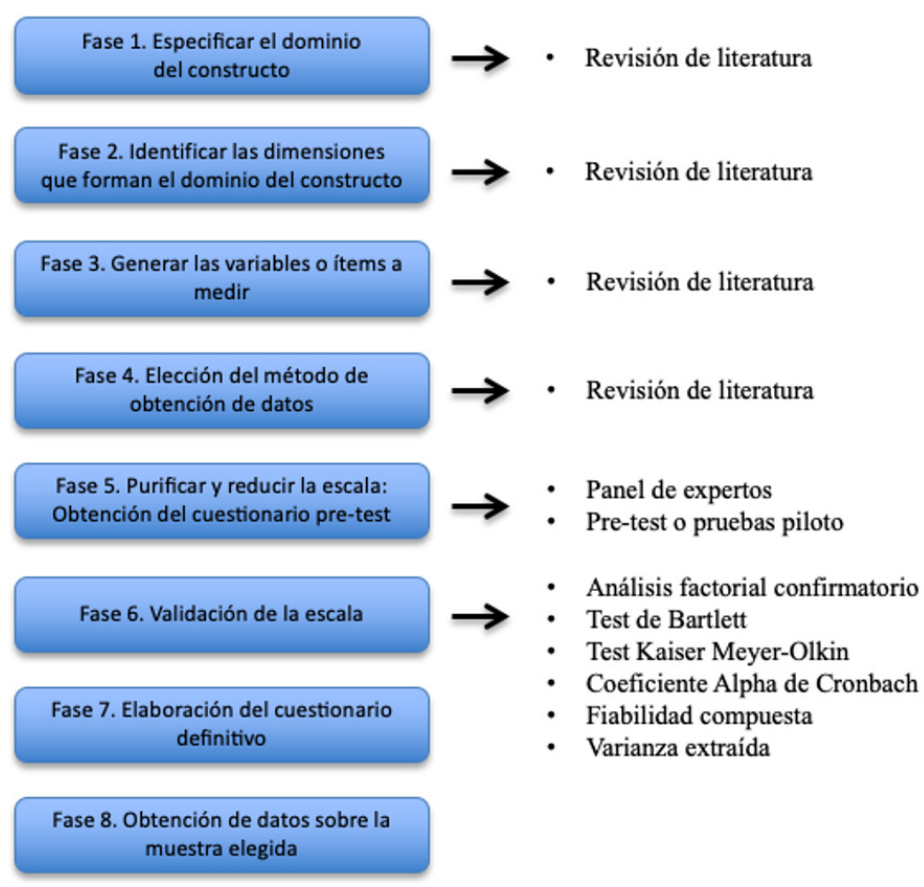

Fuente: Adaptación del modelo planteado por Camisón y Cruz (2008).

\section{METODOLOGÍA PARA EL ANÁLISIS DE LOS DATOS DE LA ESCALA DE MEDICIÓN}

Con el fin de facilitar el entendimiento sobre las respuestas de los encuestados, se le aplica un puntaje entre 1 y 5 a cada posibilidad de respuesta de la escala Likert, de forma tal que, dicho puntaje pudiera servir para ser utilizado posteriormente en la construcción de un indicador de cada variable. 
Se hace necesario destacar, que algunos de los ítems del cuestionario plantean situaciones positivas cuya frecuencia se desea alta para determinar el grado de adopción de un servicio financiero innovador, por ejemplo: "Utilizar servicios financieros innovadores, hace que yo tenga un mejor desempeño en mi trabajo o mi vida personal." Por otra parte, algunos ítems se refieren a situaciones negativas, las cuales pueden generar que las personas tengan una resistencia hacia la adopción de un servicio financiero innovador, lo cual, se espera que no ocurra, pues la innovación financiera es fundamental para el desarrollo económico de los países. Un ejemplo de ítem redactado negativamente es: "Considero que los servicios financieros innovadores me podrían generar algún tipo de daño o riesgo al utilizarlos."

Para efectos de mantener la consistencia de los datos, los puntajes asignados a cada respuesta fueron recodificados, realizando el ajuste de modo que los ítems con redacción en negativo de una variable, tuvieran invertido su puntaje, para minizar su efecto, en el proceso de adopción de un servicio financiero innovador. Es decir, que de esta manera, el puntaje total de cada variable, refleja ya no una alta o baja frecuencia de una situación, sino más bien, una percepción positiva hacia la toma de decisiones para adoptar un servicio financiero innovador. Por lo tanto, un ítem como el siguiente: "Los servicios financieros tradicionales que me ofrecen en el mercado, me generan desconfianza.", obtiene un puntaje de 5 puntos en la opción "Totalmente en desacuerdo" y 1 punto en la opción "Totalmente de acuerdo", garantizando así que, el menor puntaje sea el que no contribuye a la adopción de un servicio financiero innovador. De igual manera, un ítem como: "Me parece que los servicios financieros innovadores son fáciles de entender y fáciles de usar.", recibe una puntuación de 5 puntos en la opción "Totalmente de acuerdo", pues, de acuerdo a la Teoría de la Difusión de las Innovaciones mencionada anteriormente, la facilidad de entendimiento y la facilidad de uso, son elementos que contribuyen con la adopción de la innovación. Así entonces, utilizando el mismo ítem, la opción "Totalmente en desacuerdo", recibe un puntaje de 1 punto, debido a que al escoger esta opción, el encuestado estaría indicando que el proceso de adopción de la innovación no tiene relación con la facilidad y el entendimiento sobre la innovación. Se entiende por supuesto, que luego de procesar los datos electrónicamente, los puntajes altos en las variables, reflejan su importancia en la toma de decisiones para adoptar un servicio financiero innovador, mientras que los puntajes bajos, reflejan lo contrario. 
De igual manera, se somete el cuestionario a la validación por medio del sistema Stata en su versión 15C, mediante el cual, se lleva a cabo la medición del índice Kaiser Meyer-Olkin (KMO). Los valores de $\mathrm{KMO}$ entre 0.5 y 1 indican que es apropiado aplicar el análisis factorial a la matriz de datos bajo estudio (Montoya, 2007).

Así mismo, se aplica el Test de Esfericidad de Bartlett, el cual se utiliza para probar la hipótesis nula que afirma que las variables no están correlacionadas en la población. Es decir, comprueba si la matriz de correlaciones es una matriz de identidad. Se puede dar como válidos aquellos resultados que nos presenten un valor elevado del test y cuya fiabilidad sea menor a 0.05 . En este caso se rechaza la hipótesis nula y se continúa con el análisis.

Luego de llevarse a cabo estos cálculos estadísticos, se somete la escala al Análisis Factorial Confirmatorio, el cual es una técnica de reducción de datos que sirve para encontrar grupos homogéneos de variables a partir de un conjunto numeroso de variables. Los grupos homogéneos se forman con las variables que correlacionan mucho entre sí y procurando, inicialmente, que unos grupos sean independientes de otros. Cuando se recogen un gran número de variables de forma simultánea (por ejemplo, en un cuestionario de satisfacción laboral) se puede estar interesado en averiguar si las preguntas del cuestionario se agrupan de alguna forma característica. Aplicando un análisis factorial a las respuestas de los sujetos se pueden encontrar grupos de variables con significado común y conseguir de este modo reducir el número de dimensiones necesarias para explicar las respuestas de los sujetos.

El análisis factorial es, por tanto, una técnica de reducción de la dimensionalidad de los datos. Su propósito último consiste en buscar el número mínimo de dimensiones capaces de explicar el máximo de información contenida en los datos.

De igual manera, y para determinar la fiabilidad de la escala, se aplica a los datos de los planes piloto, la medida del Alpha de Cronbach que es un coeficiente que sirve para medir la fiabilidad de una escala de medida. Luego de aplicar esta medida, se verifica que el coeficiente de fiabilidad (Scale reliability coefficient) presente valores menores a 0.7, no obstante valores por encima de 0.6 son aceptados por considerarse que el estudio es de naturaleza exploratoria. 
Así mismo, se realiza el cálculo de la fiabilidad compuesta, la cual debe superar un mínimo de 0.7 y la varianza extraída que de igual manera debe superar el rango mínimo de 0.5. Así entonces, luego de aplicar todo el procedimiento anteriormente explicado, se procede a concluir que la escala queda totalmente validada.

Finalmente, el análisis de estos ítems se realiza llevando a cabo la distribución porcentual (promedio simple) de las respuestas en cada una de las categorías. De esta manera se identifican las dimensiones que fueron evaluadas en forma más positiva y más negativa, determinando con esta metodología, la importancia relativa de cada una de dichas dimensiones, y por ende de las variables a las que pertenecen, sobre el proceso de toma de decisiones de las personas para la adopción o no, de un servicio financiero innovador.

\section{ÍNDICES DE PERCEPCIÓN EN LA ADOPCIÓN DE SERVICIOS FINANCIEROS INNOVADORES, SEGÚN LAS DIMENSIONES DE LA ESCALA}

Los índices son calculados de acuerdo a las veintidós dimensiones que posee la escala, mismas que se agrupan en 8 variables, de las cuales, una variable posee datos relacionados con los factores demográficos, como la edad, el género, el nivel educacional, entre otros, que no se toman en cuenta en la aplicación de la metodología por puntajes.

Estas variables, se analizan por medio de cálculos estadísticos del sistema Stata, versión 15C, cuantificando sus porcentajes, de acuerdo a los resultados de las respuestas del cuestionario. Los índices se calculan de la siguiente manera: 
Tabla 2. Metodología de cálculo de índices.

(De acuerdo con su fórmula de cálculo)

\begin{tabular}{|c|c|c|}
\hline Variable & $\begin{array}{l}\text { Fórmula de } \\
\text { cálculo }\end{array}$ & $\begin{array}{l}\text { Descripción de la } \\
\text { metodologia de } \\
\text { cálculo }\end{array}$ \\
\hline $\begin{array}{l}\text { Conocimiento } \\
\text { acerca de la inno- } \\
\text { vación. }\end{array}$ & $\overline{x_{1}}=\frac{\sum_{i=1}^{27} x_{1 i}}{27}$ & $\begin{array}{l}\text { Promedio simple } \\
\text { de la escala de co- } \\
\text { nocimiento acerca } \\
\text { de un servicio fi- } \\
\text { nanciero innova- } \\
\text { dor. }\end{array}$ \\
\hline $\begin{array}{l}\text { Percepción de uti- } \\
\text { lidad de uso. }\end{array}$ & $\overline{x_{2}}=$ & $\begin{array}{l}\text { Promedio simple } \\
\text { de la escala de } \\
\text { percepción de la } \\
\text { utilidad de uso de } \\
\text { un servicio finan- } \\
\text { ciero innovador. }\end{array}$ \\
\hline $\begin{array}{l}\text { Percepción de fa- } \\
\text { cilidad de uso. }\end{array}$ & $\overline{x_{3}}=$ & $\begin{array}{l}\text { Promedio simple } \\
\text { de la escala de } \\
\text { percepción de la } \\
\text { facilidad de uso de } \\
\text { un servicio finan- } \\
\text { ciero innovador. }\end{array}$ \\
\hline $\begin{array}{l}\text { Actitud hacia el } \\
\text { uso. }\end{array}$ & $\overline{x_{4}}=$ & $\begin{array}{l}\text { Promedio simple } \\
\text { de la escala de la } \\
\text { actitud de uso de } \\
\text { un servicio finan- } \\
\text { ciero innovador. }\end{array}$ \\
\hline Intención de uso. & $\overline{x_{5}}=$ & $\begin{array}{l}\text { Promedio simple } \\
\text { de la escala de la } \\
\text { intención de uso } \\
\text { de un servicio fi- } \\
\text { nanciero innova- } \\
\text { dor. }\end{array}$ \\
\hline $\begin{array}{l}\text { Factores demo- } \\
\text { gráficos. }\end{array}$ & $F D=$ & $\begin{array}{l}\text { Promedio simple } \\
\text { de las respuestas } \\
\text { obtenidas sobre } \\
\text { las preguntas rela- } \\
\text { cionadas a facto- } \\
\text { res demográficos. }\end{array}$ \\
\hline $\begin{array}{l}\text { Factores sociocul- } \\
\text { turales. }\end{array}$ & $F C=$ & $\begin{array}{l}\text { Promedio simple } \\
\text { de la escala rela- } \\
\text { cionada con los } \\
\text { factores sociocul- } \\
\text { turales. }\end{array}$ \\
\hline $\begin{array}{l}\text { Toma de decisio- } \\
\text { nes. }\end{array}$ & $\overline{x_{8}}=\underline{\sum_{i=1}^{6} x_{a i}}$ & $\begin{array}{l}\text { Promedio simple } \\
\text { de la escala de } \\
\text { toma de decisión } \\
\text { para adoptar un } \\
\text { servicio financiero } \\
\text { innovador. }\end{array}$ \\
\hline
\end{tabular}

Fuente: Elaboración propia. 


\section{COMPARACIÓN DE ÍNDICES}

Con el fin primordial de establecer de una manera más clara, la incidencia e importancia que tienen tanto los factores demográficos como los factores socioculturales, así como todos aquellos aspectos que, según la teoría, inciden en la adopción de la innovación, se realizan análisis sobre datos estadísticos y se analizan los índices de correlación entre dichos factores, utilizando el sistema Stata, en su versión 15C. También se llevan a cabo regresiones para determinar el efecto de las variables independientes con la variable dependiente.

\section{METODOLOGÍA DE APLICACIÓN DEL PLAN PILOTO PARA POENR A PRUEBA EL INSTRUMENTO DE MEDICIÓN}

a) Aplicación del plan piloto etapa 1

El plan piloto, se aplica en dos etapas, la primera se lleva a cabo en un lapso de una semana específicamente del 01 de noviembre de 2019 al día 05 del mismo mes. En esta primera aplicación del plan piloto, se escogen al azar 20 personas del total de la población de estudio y se les aplica el cuestionario de forma virtual utilizando el sistema Google Forms, mismo que hace envío del cuestionario vía correo electrónico a los encuestados.

Una vez finalizada esta primera etapa del plan piloto, se procedió a implementar las mejoras en el cuestionario incorporando al final del instrumento de medición, un ítem de respuesta abierta en donde se le permite al encuestado opinar sobre los factores que influyen según su criterio para llevar a cabo el proceso de adopción de servicios financieros innovadores. Finalmente, se mejoró la redacción de algunos ítems para que su contenido fuera más conciso y entendible, sin llegar a modificar su significado.

b) Aplicación del plan piloto etapa 2

Una vez implementados los ajustes al instrumento de investigación, se procede a aplicar el cuestionario a una segunda submuestra, la cual de igual manera es escogida al azar, pero en esta ocasión se utiliza una submuestra de 280 personas que corresponde a un $72,92 \%$ del total de la muestra requerida para el estudio que es de 384 personas. 
Esta aplicación de la segunda etapa del plan piloto, se lleva a cabo utilizando también la herramienta Google Forms, por medio de la cual, se envían 280 invitaciones para completar el cuestionario vía Internet. La aplicación de esta etapa del plan piloto, se realiza del día 10 de noviembre de 2019 y finaliza el día 24 del mismo mes, recolectando un total de 83 cuestionarios, que equivalen a un $29,64 \%$, del total de las invitaciones enviadas, y un $21,61 \%$ de la muestra total requerida para la investigación que es de 384 personas.

En esta aplicación de la segunda etapa del plan piloto, se determinó que los encuestados tuvieron una mejor aceptación del instrumento, completando todas las respuestas. Además, se pudo determinar, que los tiempos requeridos por los encuestados para responder el cuestionario se mantuvieron dentro del parámetro de 15 minutos, siendo la media un tiempo de 12 minutos. 


\section{ANÁLISIS E INTERPRETACIÓN DE RESULTADOS}

Con el fin de entender de mejor manera el efecto de cada variable, se analiza cada una de ellas por separado, no obstante todas las variables que no son demográficas (edad, género, nivel educacional, nivel económico, profesión y estado civil) se consideran variables socioculturales. Seguidamente se muestran los principales datos estadisticos de la muestra:

Figura 5. Datos estadísticos de la muestra.
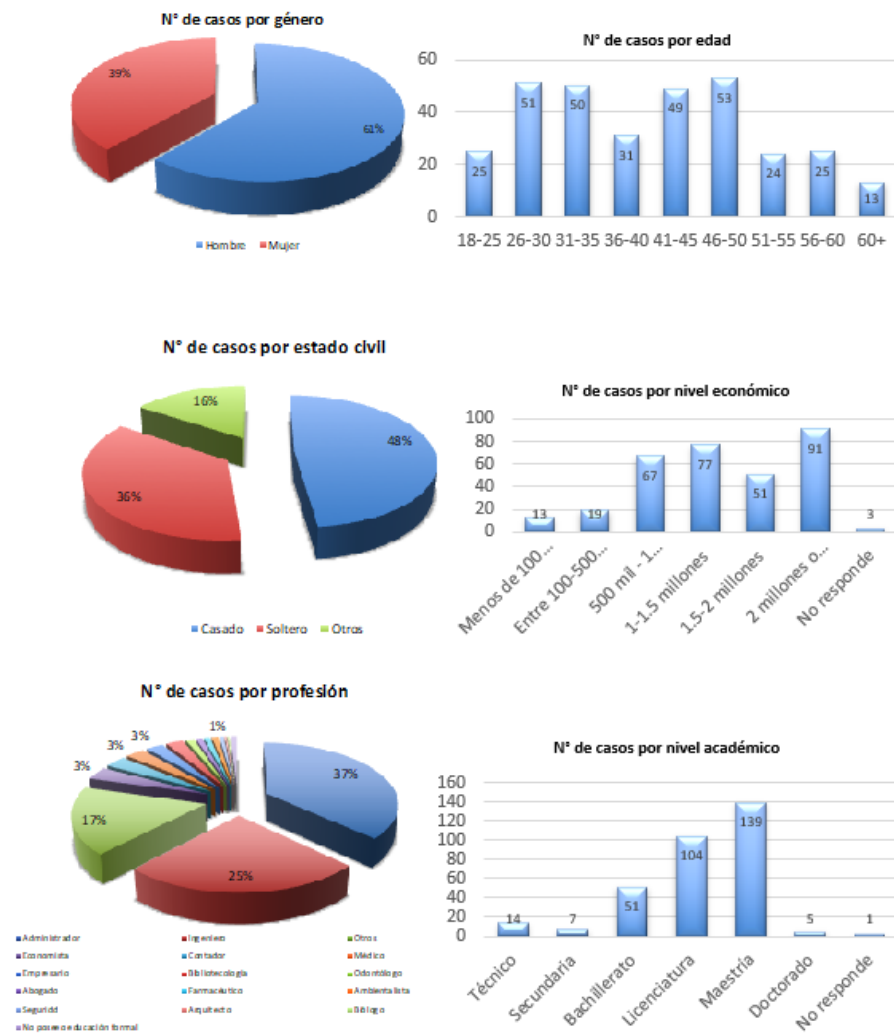

Fuente: Elaboración propia. 
Así mismo, y luego de la aplicación de la metodología de puntajes, se determina que la principal variable que incide en la toma de decisiones de las personas para adoptar un servicio financiero innovador es la "Percepción de Utilidad", dentro de la cual, su principal dimensión es la relacionada con los beneficios que brindan dichos servicios financieros. De igual manera, la variable "Conocimiento" es muy importante, pues se conforma como la base para que las personas pueden percibir la utilidad de uso de los servicios financieros innovadores.

Tabla 3. Resultados de la metodología de puntajes.

\begin{tabular}{|l|c|l|}
\hline \multicolumn{1}{|c|}{ Variable } & Por centaje & \multicolumn{1}{c|}{ Dimensión } \\
\hline Percepción de utilidad de uso & $24,92 \%$ & Beneficio \\
\hline Conocimiento de la innovación & $23,82 \%$ & Ventaja relativa \\
\hline Factores socioculturales & $22,57 \%$ & Aversión al riesgo \\
\hline Percepción de facilidad de uso & $10,81 \%$ & Demostrabilidad \\
\hline Actitud hacia el uso & $10,00 \%$ & Voluntariedad \\
\hline Intensión de uso & $4,15 \%$ & Intensión de uso \\
\hline Toma de decisiones & $3,72 \%$ & Influencia grupal \\
\hline
\end{tabular}

Fuente: Elaboración propia.

Para comprobar aún más los resultados encontrados con la utilización de la metodología de puntajes, se aplica la regresión logística, que es una técnica muy utilizada en estadística, misma que es un tipo de análisis de regresión utilizado para predecir el resultado de una variable categórica (una variable que puede adoptar un número limitado de categorías) en función de las variables independientes o predictoras. Para el caso de esta investigación, la variable dependiente es la adopción de servicios financieros innovadores, misma que toma solo dos valores, a saber: Si adopta y No adopta. La regresión logística se aplica para analizar la tendencia de los encuestados antes y durante la pandemia del COVID-19. 
Tabla 4. Cálculo de la regresión logística.

(Antes y durante la pandemia del COVID-19)

\begin{tabular}{|c|c|c|}
\hline & ANTES DE COVID-19 & \\
\hline Variable & Nivel de significancia & Odds Ratio \\
\hline Percepción de Utilidad & 0,012 & 2,65 \\
\hline & & \\
\hline Variable & DESPUÉS DE COVID-19 & \\
\hline Percepción de Utilidad & Nivel de significancia & Odds Ratio \\
\hline
\end{tabular}

Fuente: Elaboración propia.

La regresión logística muestra que existe una significancia antes y después de la pandemia del COVID-19 ( $P=0,012 ; P=0,043$, respectivamente) para la variable "Percepción de utilidad", lo cual la ratifica como la variable más relevante en el proceso de adopción de un servicio financiero innovador, pues tanto en la metodología de puntajes, como en la regresión logística para los resultados de adopción de servicios financieros de los encuestados hace un año, así como los resultados de la regresión logística para las variables en estudio al día de hoy, dicha variable sigue mostrando significancia.

Así entonces, por cada punto que aumente la percepción de utilidad, aumenta 2,65 veces la propensión de adoptar, misma que disminuye a 1,98 durante la pandemia de COVID-19. No se encuentra suficiente evidencia estadística para determinar niveles de significancia en todos los factores demográficos y socioculturales adicionales, que permitan determinar algún efecto moderador en el proceso de toma de decisiones para adoptar un servicio financiero innovador.

Dado lo anterior, se someten a comprobación las hipótesis planeadas en la investigación, resumiendose su resultado de la siguiente manera: 
Tabla 5. Comprobación de hipótesis.

\begin{tabular}{|c|c|c|c|}
\hline Número & Hipótesis & Resultado & Significancia \\
\hline H1 & $\begin{array}{l}\text { Las personas que poseen una mayor aversión al riesgo } \\
\text { utilizan menos los servicios financieros nuevos e } \\
\text { innovadores. }\end{array}$ & No se rechaza & ( $p$ value $>0,05$ ) \\
\hline H2 & $\begin{array}{l}\text { Las personas son más propensas a tomar la decisión de } \\
\text { aceptar y adoptar servicios financieros innovadores según } \\
\text { su grado de educación académica sea mayor. }\end{array}$ & No se rechaza & ( $p$ value $>0,05$ ) \\
\hline H3 & $\begin{array}{l}\text { Elementos culturales de la familia, inciden directamente } \\
\text { en la toma de decisiones de las personas para adoptar } \\
\text { servicios financieros innovadores. }\end{array}$ & No se rechaza & ( $p$ value $>0,05$ ) \\
\hline $\mathrm{H} 4$ & $\begin{array}{l}\text { Las personas con más edad, tienen menor tendencia a } \\
\text { utilizar servicios financieros nuevos e innovadores. }\end{array}$ & No se rechaza & ( $p$ value $>0,05$ ) \\
\hline H5 & $\begin{array}{l}\text { Personas con mayor experiencia o capacitación en el uso } \\
\text { de servicios financieros, poseen mayor nivel de aceptación } \\
\text { y adopción de servicios financieros innovadores. }\end{array}$ & No se rechaza & ( $p$ value $>0,05$ ) \\
\hline H6 & $\begin{array}{l}\text { Las personas con niveles económicos menores tienen } \\
\text { menos tendencia a utilizar servicios financieros nuevos e } \\
\text { innovadores. }\end{array}$ & No se rechaza & ( $p$ value $>0,05$ ) \\
\hline $\mathrm{H7}$ & $\begin{array}{l}\text { El género en las personas no es un factor determinante en } \\
\text { el proceso de la toma de decisión para adoptar servicios } \\
\text { financieros innovadores. }\end{array}$ & No se rechaza & ( $p$ value $>0,05$ ) \\
\hline
\end{tabular}

Fuente: Elaboración propia.

De acuerdo con lo anterior, no se encontró suficiente evidencia estadística para rechazar las hipótesis planteadas. 


\section{CONCLUSIONES}

Este estudio contribuye a las teorías utilizadas, en aspectos determinantes como el hecho de que las teorías que han servido de base para esta investigación no toman en cuenta generaciones más recientes y sus comportamientos. Esta investigación contrasta de manera efectiva las preferencias y comportamientos de varias generaciones y los aspectos culturales que inciden en ellas, pudiendo los resultados, servir para diseñar un modelo de negocio para un servicio financiero innovador exitoso, pues desde hace muchos años, nuestro país evidencia una escacez de servicios financieros innovadores, y más bien, las historias de fracaso han sido evidentes, trayendo con esto, un incremento en la aversión al riesgo de las personas y por ende una desaceleración en el crecimiento o incluoso, un decrecimiento en las operaciones de las instituciones financieras, afectando también el desarrollo del país.

De igual manera, esta investigación contribuye al desarrollo del sector financiero tanto a nivel local como internacional, pues genera información sumamente valiosa para la toma de decisiones de la alta gerencia de las instituciones financieras y garantiza en cierta medida, una base sólida para determinar los cursos de acción y los modelos de negocio a seguir para fortalecer la participación exitosa de las personas en el negocio financiero, y las estrategias que permitan maximizar las ventajas competitivas de cada uno, en búsqueda de un bienestar económico compartido entre las instituciones financieras y los clientes, mismo que debe ser sostenible a través de los años, pues no se trata de que los clientes deban amoldarse a servicios financieros mal diseñados que a la postre, terminan generando dificultades económicas y de control que afectan el bienestar económico y social de la población.

A pesar de que factores como la aversión al riesgo, la experiencia y familia presentaron ciertas variaciones en sus promedios, no existe suficiente evidencia estadística para afirmar que los factores socioculturales de las personas inciden directamente en el proceso de toma de decisiones para adoptar servicios financieros innovadores.

De igual manera, la investigación no presenta en cuanto a la adopción de servicios financieros innovadores por parte de las personas, niveles de significancia que permitan tener evidencia estadística suficiente para identificar efectos mediadores por parte de las variables demográficas. Es decir, que elementos como la edad, el nivel educativo, entre otros, parecieran no ser tan relevantes en el proceso de adopción de servicios financieros innovadores. 
La única variable que muestra niveles de significancia es la "Percepción de Utilidad", lo que brinda evidencia estadística suficiente para identificar que esta variable es la más importante en el proceso de toma de decisiones de las personas para adoptar servicios financieros y en donde los beneficios que ofrecen los servicios financieros innovadores, son fundamentales en el proceso de adopción, pues se conforman como la dimensión más importante de la "Percepción de Utilidad".

A pesar de que la propensión de adoptar servicios financieros innovadores disminuyó por parte de los encuestados durante la pandemia del COVID-19, no se encontró suficiente evidencia estadística para determinar que los factores socioculturales y demográficos de las personas en estudio, sufrieron algún cambio que afectara su proceso de toma de decisiones para adoptar servicios financieros innovadores durante la época de pandemia. No obstante, se encontró evidencia estadística de que la "Percepción de Utilidad", mostró una disminución en su nivel de significancia y por ende en su nivel de propensión a la adopción (Odds Ratio), es decir, que las personas se han vuelto más cautelosas ante la crisis sanitaria.

Uno de los principales hallazgos, se centra en el hecho de que la variable "Conocimiento" podría ser fundamental para que las personas obtengan confianza en el uso de un servicio financiero innovador, pues por medio del conocimiento que pueden percibir la utilidad de uso.

Elementos como la influencia familiar, incluso la influencia de amigos o grupos sociales, no muestran ninguna tendencia para incidir en la toma de decisiones de las personas en cuanto a la adopción de servicios financieros innovadores. Por lo tanto, se puede concluir que los factores socioculturales y demográficos de las personas, a pesar de su importancia, no muestran indicios de ejercer una moderación en el proceso de toma de decisiones para adoptar servicios financieros innovadores, pues no hay evidencia estadística suficiente para afirmarlo.

Dado que luego de llevarse a cabo una exhaustiva revisión del estado del arte y la búsqueda de información por medio de fuentes académicas, bases de datos, repositorios y de otras fuentes de gran prestigio e importancia a nivel internacional desde el punto de vista académico y científico, no se pudo encontrar evidencia de investigaciones relacionadas con los factores que inciden en la adopción de servicios financieros por parte de las personas, por lo que esta investigación da origen a lo que se puede denominar como la Teoría de la Adopción Financiera (Financial Adoption Theory). 
De todo lo anterior, se sugiere que a futuro puedan llevarse a cabo las siguientes líneas de investigación: es importante realizar el estudio en zonas rurales del país e incluso a nivel internacional, para contrastar los factores socioculturales y demográficos en distintas culturas, pues es claro que las sociedades cambian y se adaptan según su historia y sus raíces. Así mismo, es importante realizar la investigación de manera más presencial para evitar la posible resistencia al uso de mecanismos tecnológicos de recolección de datos, llegando de esta manera a todos los estratos sociales del país y poder conocer la percepción de las distintas comunidades a nivel nacional e incluso internacional.

Finalmente, es importrante realizar la investigación en tiempos que no sean de crisis económica y sanitaria como las que se viven actualmente por la emergencia mundial provocada por el COVID-19, pues es claro que principalmente la pandemia del Coronavirus, ha impactado social, económica y psicológicamente a todas las personas, indistintamente de sus factores socioculturales y demográficos. 


\section{BIBLIOGRAFÍA}

Ajzen, I. (1985). From intentions to actions: A theory of planned behaivor. En J. Kuhl y J. Beckmann (Eds.), Action control: from cognition to behavior (pp. 11-39). Heidelberg, Germany: Springer.

Ajzen, I. (1991). The theory of planned behaviour. Organizational Behaviour and Human Decision Processes, 50(2), 179-211.

Ajzen, I., 2005. Attitudes, Personality, and Behavior. McGraw-Hill Education, UK).

Ajzen, I., 2012. The theory of planned behavior. In: Lange, P.A.M., Kruglanski, A.W., Higgins, E.T. (Eds.), Handbook of Theories of Social Psychology 1. Sage, London, UK, pp. 438-459.

Aldy, J., Hrubovcak, J., y Vasavada, J. (1998). The role of technology in sustaining agriculture and the environment. Ecological Econom, 26, 81-96.

Anderson, Theresa D. (2011). Beyond eureka moments: supporting the invisible work of creativity and innovation. Information Research, 16(1). Recuperado de http://InformationR.net/ir/16-1/paper471.html

Azadi, Y., Yazdanpanah, M., Mahmoudi, H., 2019. Understanding smallholder farmers' adaptation behaviors through climate change beliefs, risk perception, trust, and psychological distance: evidence from wheat growers in Iran. J. Environ. Manag. 250, 109456.

Barrett, C. A., Pas, E. T. y Lindstrom, S. (abril, 2020). A cost analysis of the innovation-decision process of an evidence-based practice in schools. School Mental Health. Recuperado de https://doi.org/10.1007/s12310-020-09372-z

Barrutieta, L. H. (2011). Influencia de la emoción en la toma de decisión: implicaciones en la producción de inteligencia. Inteligencia y Seguridad: Revista de Análisis y Prospectiva, 10, 103-122.

Bechara, A., Damasio, H., y Damasio, A. (marzo, 2000). Emotion, decision making and the orbitofrontal cortex. Cerebral Cortex, 10(3), 295-307. 
Bekkering E, Hutchison D (2009) A follow-up study of using remote desktop applications in education. Inf Syst Educ J 7(55):13

Bird, E.L., Panter, J., Baker, G., Jones, T., Ogilvie, D., iConnect, Consortium, 2018. Predicting walking and cycling behaviour change using an extended Theory of Planned Behaviour. J. Transport Health 10, 11e27.

Boholm, A. (2003). The cultural nature of risk: can there be an anthropology of uncertainty? Ethnos. Journal of Anthropology, 68(2), 159-178.

Bower, G. (1981). Mood and memory. American Psychologist, 36 (2), 129-148.

Brunner, T. A., Van der Horst, K., \& Siegrist, M. (2010). Convenience food products. Drivers for consumption. Appetite, 55(3), 498-506.

Camisón, C., \& Cruz, S. (2008). La medición del desempeño organizativo desde una perspectiva estratégica: creación de un instrumento de medida. Revista Europea de Dirección y Economía de la Empresa, 17(1), 79-102.

Candel, M. J. (2001). Consumers' convenience orientation towards meal preparation: Conceptualization and measurement. Appetite, 36(1), 15-28.

Cefis, E., y Marsili, O. (2006). Survivor: the role of innovation in firms' survival. Research Policy, 35(5), 626-641. doi:10.1016/j. respol.2006.02.006

Chen, Y., Chen, C., Lin, Y., y Yeh, R. (2007). Predicting college student' use of e-learning systems: an attempt to extend technology acceptance model. En Proceedings of PACIS, Paper 121.

Chen, M.F., 2017. Modeling an extended theory of planned behavior model to predict intention to take precautions to avoid consuming food with additives. Food Qual. Prefer. 58, 24e33.

Cherfi. S. (2011). La culture familiale et la decisión d'achat: cas de la famille française d'origine maghrébine. Revue des Sciences de Gestion, (252), 8997. 
Contini, C., Boncinelli, F., Marone, E., Scozzafava, G. y Casini, L. (setiembre, 2020). Drivers of plant-based convenience foods consumption: results of a multicomponent extension of the theory of planned behavior. Food Quality and Preference, 84, 1-9. Recuperado de https://doi.org/10.1016/j.foodqual.2020.103931

Cordano, M., Frieze, I.H., 2000. Pollution reduction preferences of U.S. environmental managers: applying Ajzen'S theory of planned behavior. Acad. Manag. J. 43 (4), 627e641.

Cosentino, A., Azzollini, Depaula, P., y Castillo, S. (2016). Toma de decisión según racionalidad/afectividad, entrenamiento y saturación cultural en situaciones multiculturales: un estudio experimental con soldados para la paz. Interdisciplinaria, 33(2), 283-298.

Davis, F. D. (1989). Perceived Usefulness, Perceived Ease of Use, and User Acceptance of Information Technology. MIS Quarterly, 13(3), 319-340. doi: 10.2307/249008.

Davis, F. D., Bagozzi, R. P., y Warshaw, P. R. (1989). User acceptance of computer technology: A comparison of two theoretical models. Management Science, 36(8), 982-1003.

Davis, L.E., Ajzen, I., Saunders, J., Williams, T., 2002. The decision of African American students to complete high school: an application of the theory of planned behavior. J. Educ. Psychol. 94 (4), 810.

De Boer, M., McCarthy, M., Cowan, C., \& Ryan, I. (2004). The influence of lifestyle characteristics and beliefs about convenience food on the demand for convenience foods in the Irish market. Food Quality and Preference, 15(2), 155-165.

DeSteno, D., Petty, R., Rucker, D., y Wegener, D. (2000). Beyond valence in the perception of likelihood: the role of emotion specificity. Journal of Personality and Social Psychology, 78 (3), 397-416.

Druckman, J. y McDermott, R. (2008). Emotion and the framing of risky choice. Political Behavior, 30 (3), 297-321. 
Dunn, K. I., Mohr, P., Wilson, C. J., \& Wittert, G. A. (2011). Determinants of fast-food consumption. An application of the theory of planned behaviour. Appetite, 57(2), 349-357.

Faiers, A., Cook, M., Neame, C., 2007. Towards a contemporary approach for understanding consumer behaviour in the context of domestic energy use. Energy Policy 35 (8), 4381-4390.

Forero, C., Rojas, G., y Arguelles, J. (2013). Capital social y capital financiero en la adopción de tecnologías ganaderas en zonas rurales alto andinas de Colombia. Revista Corpoica Ciencia y Tecnología Agropecuaria. 14(2), 149-163. framework. Recuperado de http://lem.cnrs.fr/Portals/2/actus/DP_200824.pdf.

Greenhalgh, T., Robert, G., MacFarlane, F., Bate, P., \& Kyriakidou, O. (2004). Di usion of innovations in service organizations: Sys- tematic review and recommendations. The Milbank Quarterly:AMultidisciplinary Journal of Population Health and Heath Policy, 82, 581-629. https://doi.org/10.1111/j.0887-378X.2004.00325.x.

Hardeman, W., Johnston, M., Johnston, D.W., Bonetti, D., Wareham, N.J., Kinmonth, A.L., 2002. Application of the theory of planned behaviour in behaviour change in- terventions: a systematic review. Psychol. Health 17 (2), 123-158.

Hayton, J. C., y Kelley, D. J. (2006). A competency-based framework for promoting corporate entrepreneurship. Human Resource Management, 45(3), 407-427. doi:10.1002/hrm.20118

Heinze, K. L. y Heinze, J. E. (junio, 2020). Individual innovation adoption and the role of organizational culture. Review of Managerial Science, 14, 561-586. Recuperado de https://link.springer.com/article/10.1007/s11846-018-0300-5

Hernández, R., Fernández, C., y Batista, P. (2010) Fundamentos de la metodología de la investigación. México. Editorial McGraw-Hill.

Johnson, E., y Amos, T. (1983). Affect, generalization, and the perception of risk. Journal of Personality and Social Psychology, 45(1), 20-31. 
Jolibert, A., y Valette-Florence, P. (1985). Un essai empirique de clarification des approches des styles de vie. S.I.: s.e.

Judge, M., Warren-Myers, G., Paladino, A., 2019. Using the theory of planned behaviour to predict intentions to purchase sustainable housing. J. Clean. Prod. 215, 259e267.

Kaffashi, S., Shamsudin, M.N., 2019. Transforming to a low carbon society; an extended theory of planned behaviour of Malaysian citizens. J. Clean. Prod. 235, $1255 \mathrm{e} 1264$.

Kim, J., y Forsythe, S. (2008). Adoption of virtual try-on technology for online apparel shopping. Journal of Interactive Marketing, 22(2), 45-59.

Lapple, D., Kelley, H., 2013. Understanding the uptake of organic farming: accounting for heterogeneities among Irish farmers. Ecol. Econ. 88, 11e19.

Lerner, J., González, R., Small, D., y Fischhoff, B. (2003). Effects of fear and anger on perceived risk of terrorism: a national field experiment. Psychological Science, $14(2), 144-150$.

Liang, G., Hou, H., Chen, Q., y Hu, Z. (abril, 2020). Diffusion and adoption: an explanatory model of "question mark" and "rising star" articles. Scientometrics, 124, 219-232. Recuperado de https://link.springer.com/article/10.1007\%2 Fs11192-020-03478-6

López, L., y López, J. (2011). Los modelos de adopción de tecnologías de la información desde el paradigma actitudinal. Cuadernos EBAPE, 9(1), 176-196.

MacKuen, M., Wolak, J., Keele, L., y Marcus, G. (julio, 2005). Emotion and citizenship. En Annual Meeting of the International Society of Political Psychology. Reunión llevada a cabo en Toronto. 
Mahon, D., Cowan, C., \& McCarthy, M. (2006). The role of attitudes, subjective norm, perceived control and habit in the consumption of ready meals and takeaways in Great Britain. Food Quality and Preference, 17(6), 474-481.

Mak, T.M.W., Yu, I.K.M., Wang, L., Hsu, S.-C., Tsang, D.C.W., Li, C.N., Yeung, T.L.Y., Zhang, R., Poon, C.S., 2019. Extended theory of planned behaviour for promoting construction waste recycling in Hong Kong. Waste Manag. 83, 161e170.

Marín, Y., Ibrahim, M., Villanueva, C., Ramírez, E., y Sepúlveda, C. (2006). Los impactos de un proyecto silvopastoril en el cambio de uso de la tierra y alivio de la pobreza en el paisaje ganadero de Matigúas, Nicaragua. Agroforestería de las Américas, 45, 109-116.

Montoya, O. (2007). Aplicación del análisis factorial a la investigación de mercados. Caso de studio, 283-284.

Oliveira, T., \& Martins, M. F. (2011). Literature review of information technology adoption models at rm level. Electronic Journal of Information Systems Evaluation, 14 (1), 110.

Oliveira, T., Thomas, M., \& Espadanal, M. (2014). Assessing the determinants of cloud computing adoption: An analysis of the manufacturing and services sectors. Information.

Olsen, N. V., Sijtsema, S. J., \& Hall, G. (2010). Predicting consumer's intention to consume ready to eat meals. The role of moral attitude. Appetite, 55(3), 534-539.

Ong, C. S., y Lai, J. Y. (2006). Gender differences in perceptions and relationships among dominants of elearning acceptance. Computers in Human Behaviour, $22(5), 816-829$.

Park, N., Lee, K. M., y Cheong, P. H. (2007). University instructors' acceptance of electronic courseware: an application of the technology acceptance model. Journal of Computer-Mediated Communication, 13(1), artículo 9. 
Perri, C., Corvello, V., 2015. Smart energy consumers: An empirical investigation on the intention to adopt innovative consumption behaviour, World Academy of Science. Eng. Technol. Int. J. Psychol. Behav. Sci. 9 (9), 3030-3041.

Ponce, A. F., Mena-Mora, A., y Rottinghaus, B. (2012). El papel de la propensión al riesgo en las decisiones de voto. Política y Gobierno, 19(1), 71-99.

Ponce, M. L., y Calderón, C. A. (2016). Adopción y uso de medios sociales por jóvenes de la Costa Caribe de Colombia. Investigación y Desarrollo, 24(2), 285-306.

Rahmaninkoshkaki, M., Zarei, Y., 2018. Designing the structural equation model of agricultural entrepreneurship development in rural areas of Iran (case study: villages of marvdasht county). Int. J. Agric. Sci. Res. Technol. Ext. Educ. Syst. 8 (1), $17 \mathrm{e} 27$.

Ricci, E. C., Banterle, A., \& Stranieri, S. (2018). Trust to go green: An exploration of consumer intentions for eco-friendly convenience food. Ecological Economics, 148, 54-65.

Roca, J. C, Gagné, M. (2008). Understanding e-learning continuance intention in the workplace: a selfdetermination theory perspective. Computers in Human Behavior, 24(4), 1585-1604.

Rodríguez, T. R., y Acevedo, J. A. (2002). La cultura del empresario y su relación con la permanencia y crecimiento de las micro y pequeñas empresas en los distritos de Centro, Etla y Tlacolula en el Estado de Oaxaca. Hitos de Ciencias Económico Administrativas, 20, 7-20.

Rogers, E. M. (1962). Diffusion of Innovations (1 ed.). New York: The Free Press.

Rogers, E. M. (1983). Difussion of innovations (3 ed.). New York: The Free Press.

Rogers, E. M. (2003). Diffusion of innovations (5 ed.). New York: The Free Press.

Russell, S., Fielding, K., 2010. Water demand management research: a psychological perspective. Water Resour. Res. 46 (5). 
Saba, A., Messina, F., Turrini, A., Lumbers, M., Raats, M. M., \& Food in Later Life Project Team (2008). Older people and convenience in meal preparation: A European study on understanding their perception towards vegetable soup preparation. International Journal of Consumer Studies, 32(2), 147-156.

Salam, S., Hafeez, M., Mahmood, M. T., Iqbal, K., y Akbar, K. (2019). The dynamic relation between technology adoption, technology innovation, human capital and economy: comparison of lower-middle-income countries. Interdisciplinary Description of Complex Systems, 17(1-B), 146-161. Recuperado de https:// doi.org/10.7906/indecs.17.1.15

Salas, J. M, Leos, J.A., Sagarnaga, M., y Zavala, M.Y. (2013). Adopción de tecnologías por productores beneficiarios del programa de estímulos a la productividad ganadera (PROGAN) en México. Revista Mexicana de Ciencias Pecuarias, 4(2), 243-254.

Sánchez-Franco, M. J., Villarejo-Ramos, A. F., y Rondan-Cataluña, F. J. (2006). Male and female professors. A theoretical analysis regarding the web acceptance and use. Proceedings of the fifth European marketing trends Congress. Congreso Ilevado a cabo en Venecia, Italia.

Savari, M., Gharechaee, H. (agosto, 2020). Application of the extended theory of planned behavior to predict Iranian farmers' intention for safe use of chemical fertilizers. Journal of Cleaner Production, 263, 1-13. Recuperado de https://doi. org/10.1016/j.jclepro.2020.121512.

Scott, S., Hughes, P., Hodgkinson, I., Kraus, S., 2019. Technology adoption factors in the digitization of popular culture: Analyzing the online gambling market. Technol. Forecast. Soc. Change 148, 119717.

Schumpeter, J. A. (1942). Capitalism, socialism, and democracy. Estados Unidos: Harper and Brothers. 
Sereenonchai, S., Xu, P., Arunrat, N., y Yu, X. (2017). Diffusion and adoption behavior of environmentally friendly innovation: sharing from chinese society. The Journal of Behavioral Science, 12(2), 90-109. Recuperado de https://so06.tci-thaijo.org/index.php/IJBS/article/view/78325

Sourabh, J., Shaleen, S., Nikunj, J., y Kalyan, B. (agosto, 2020). Construction and demolition waste recycling: investigating the role of theory of planned behavior, institutional pressures and environmental consciousness. Journal of Cleaner Production, 263, 1-11. Recuperado de https://doi.org/10.1016/j.jclepro.2020.121405

Stoeva, K., Alriksson, S., 2017. Influence of recycling programmes on waste separation behaviour. Waste Manag. 68, 732e741.

Stranieri, S., Ricci, E. C., \& Banterle, A. (2017). Convenience food with environmentally- sustainable attributes: A consumer perspective. Appetite, 116, 11-20.

Talukder, M. (2014). Managing innovation adoption: from innovation to implementation. Recuperado de http://search.ebscohost.com/login.aspx?direct=true\&d$\mathrm{b}=\mathrm{e} 000 \mathrm{xw} w \& \mathrm{AN}=683468 \&$ lang=es\&site=ehost-live

Teo, M.M.M., Loosemore, M., 2001. A theory of waste behaviour in the construction industry. Construct. Manag. Econ. 19 (7), 741e751.

Van der Vegt, G., y Janssen, O. (2003). Joint impact of interdependence and group diversity on innovation. Journal of Management, 29(5), 729-751. doi:10.1016/ S0149-2063_03_00033-3.

Venkatesh, V., Morris, M. G. (2000). Why don't men ever stop to ask for directions? Gender, social influence, and their role in technology acceptance and usage behavior. MIS Quarterly, 24(1), 115-139.

Venkatesh, V., Morris, M. G., Davis, G. B., \& Davis, F. D. (2003). User acceptance of information technology: Toward a uni ed view. MIS Quarterly, 425-478. 
Wang, S., Fan, J., Zhao, D., Yang, S., Fu, Y., 2016. Predicting consumers' intention to adopt hybrid electric vehicles: using an extended version of the theory of planned behavior model. Transportation 43 (1), $123 \mathrm{e} 143$.

Walby, S. (1990). Theorizing patriarchy. Oxford: Blackwell Publishers Ltd.

Webb, D., Soutar, G.N., Mazzarol, T., Saldaris, P., 2013. Self-determination theory and consumer behavioural change: evidence from a household energy-saving behaviour study. J. Environ. Psychol. 35, 59e66.

Weber, D. M. y Kauffman, R. J. (2011). What drives global ICT adoption? Analysis and research directions. Electronic Commerce Research and Applications, 10(6), 683 $-701$.

Yadav, R., Pathak, G.S., 2016. Young consumers' intention towards buying green products in a developing nation: extending the theory of planned behavior. J. Clean. Prod. 135, 732e739.

Yazdanpanah, M., Forouzani, M., 2015. Application of the Theory of Planned Behaviour to predict Iranian students' intention to purchase organic food. J. Clean. Prod. 107, 342e352.

Yoon, C., Lim, D., y Park, C. (julio, 2020). Factors affecting adoption of smart farms: the case of Korea. Computers in Human Behavior, 108, 1-10. Recuperado de https://doi.org/10.1016/j.chb.2020.106309

Zepeda Cancino, R. M., Velasco Zebadúa, M. E., Nahed Toral, J., Hernández Garay, A., y Martínez Tinajero, J. J. (2016). Adopción de sistemas silvopastoriles y contexto sociocultural de los productores: apoyos y limitantes. Revista Mexicana de Ciencias Pecuarias, 7(4), 471-488.

Zhang, S., Zhao, J., y Tan, W. (2008). Extending TAM for online learning systems: an intrinsic motivation perspective. Tsinghua Science \& Technology, 13(3), 312-317. 


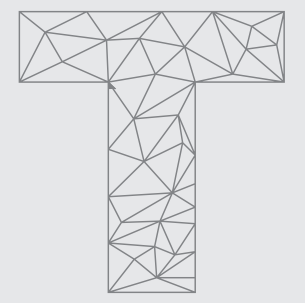

Zhu, K., Kraemer, K., \& Xu, S. (2003). Electronic business adoption by European rms: A cross-country assessment of the facilitators and inhibitors. European Journal of Information Systems, 12(4), 251-268. 